\title{
Composition Analysis with EDS; Possibilities for Life Science.
}

\author{
Meiken Falke ${ }^{1}$, A. Kaeppel ${ }^{1}$, Ralf Terborg ${ }^{1}$. \\ 1. Bruker Nano GmbH, Am Studio 2D, 12489 Berlin, Germany.
}

Energy dispersive X-ray spectroscopy (EDS) is widely established in materials science and accepted as a valuable tool for qualitative and quantitative element composition analysis. With the successful development of the silicon drift detector (SDD) technology, which provides annular multiple and large single detector arrangements, highly efficient spectroscopy for various geometric settings became possible. The aim of this contribution is to explain and evaluate options and possibilities of using this technology to solve suitable problems in life science. Biological samples and soft materials pose challenges, such as beam sensitivity, absorption effects and preparation artifacts. Therefore here, specific successful measurement conditions and respective sample types are given to help finding life science problems for which EDS may be beneficial.

The performance of EDS depends on the possible collection (solid) angle and take-off angle for the signal, on the read out electronics quality, software transparence and is additionally always linked to the electron microscopes performance and the sample geometry and preparation. EDS in an aberration corrected transmission electron microscope with a high brightness electron source can identify single atoms within minutes at a standard collection angle of $0.1 \mathrm{sr}[1]$ and within seconds if a high collection angle of e.g. $0.7 \mathrm{sr}$ is available [2]. In both cases, the single atom has to be located for the analytical experiment first. Annular multiple detector arrangements in TEM/STEM allow quantification on ppm level within hours. Samples for such type of experiments would have to be suitable for the electron dose required.

In situ experiments are another option in STEM which could be of use in life science. Successful test measurements of species in liquid were performed using a multiple detector arrangement and adapted sample holder to avoid shadowing [3].

In SEM life science samples on all scales between $\mathrm{mm}$ and micrometer can be investigated routinely and in a close to natural state if using the variable pressure mode. High collection angles ensure short measurement times, high take-off angles enable the investigation of highly topographic e.g. porous samples. Working at low voltages of $<6 \mathrm{kV}$ enables better spatial resolution but requires transparent software tools to reliably analyze the data including peak overlaps, background and absorption effects.

In figure 1 the transparent quantitative analysis tool for separating overlapping peaks is demonstrated. Not only the Ag protein labels but also the counts originating from light elements (e.g. P, S and N) and heavy Os staining and fixation can be routinely separated and quantitatively mapped. This is just one example showing, that light and heavy element distributions can be deconvoluted and mapped quantitatively. Furthermore, labelling species can be located and identified as well in the same experiment. This leads to the conclusions, that preparation technologies such as fixation and staining needed for correlative microscopies can be kept unchanged for using EDS in life science and new (or existing) different labelling species for correlative (light, fluorescence and electron) microscopy, which can be identified as well using EDS would be desirable [4]. 
References:

[1] T.C. Lovejoy et al., Appl. Phys. Lett. 100 (2012) p. 154101.

[2] R.M. Stroud et al., Microsc. Microanal. 21 (Suppl 3), 2015.

[3] E.A. Lewis et al., Chem. Commun, 2014, 50, p. 10019.

[4] M. Falke et al., Microsc. Microanal. 19 (Suppl 2) 2013.
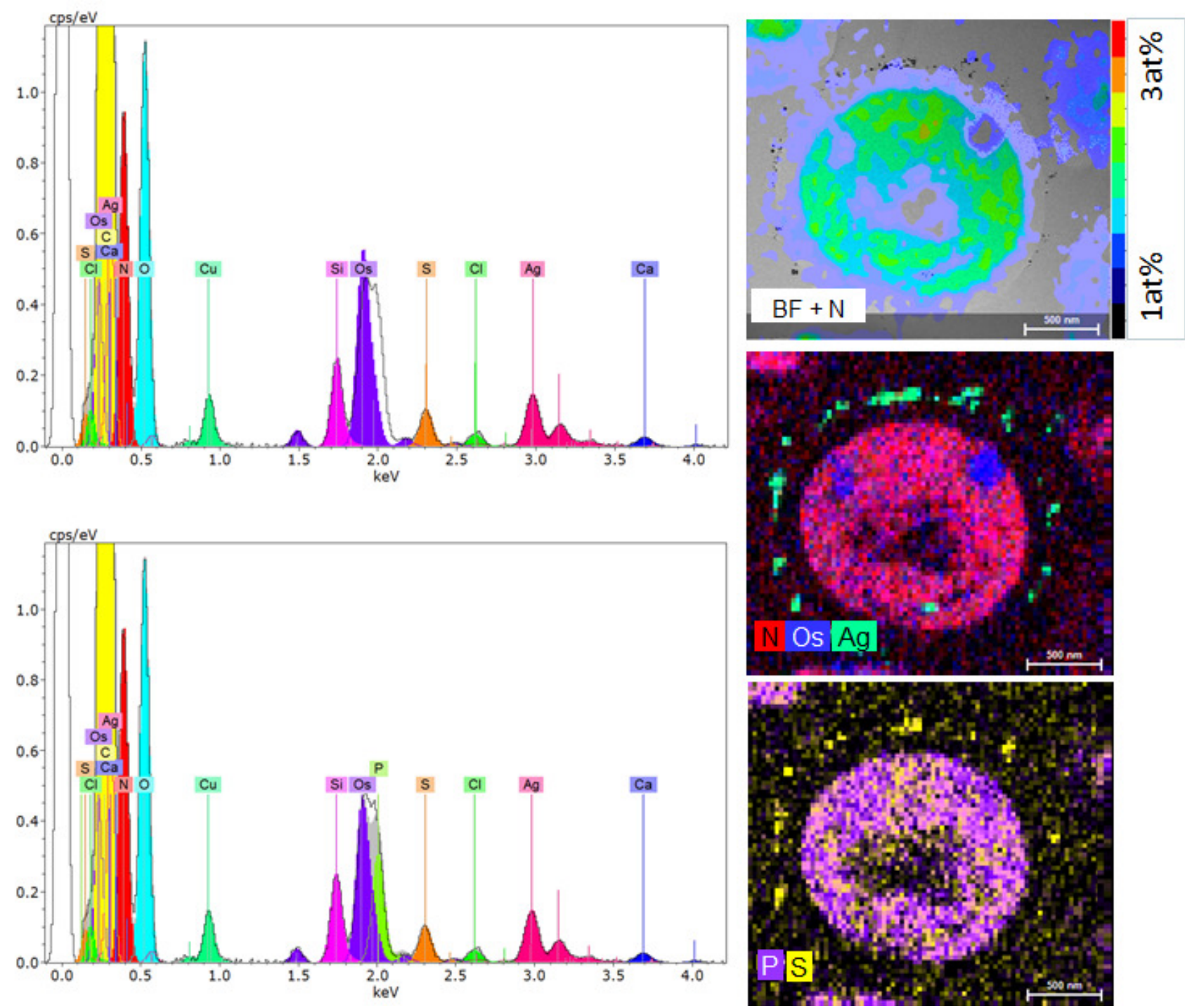

Figure 1. Thin slice of resin embedded yeast cell investigated by EDS. Upper right: Bright field (BF) image overlaid with nitrogen concentration in at\% displayed in pseudo-colors using a gentle Gaussian filter. Lower right: Element mappings of $\mathrm{N}$, Os, $\mathrm{Ag}, \mathrm{P}$ and S. Left: The measured spectra (black) with and without taking $\mathrm{P}$ into consideration (colors/green) demonstrate, that only including $\mathrm{P}$ into the quantification, the spectra can be interpreted correctly; remaining ambiguities may be due to background and absorption. Conditions: Bruker XFlash $30 \mathrm{~mm}^{2}, 0.1 \mathrm{sr}, 200 \mathrm{kV}$ STEM, $22^{\circ}$ take-off angle, $4 \mathrm{x} 4$ pixel binning, Cliff-Lorimer quantification. Data courtesy: Synergie 4 France. 